\title{
CRIBRIFORM ADENOCARCINOMA OF MINOR SALIVARY GLANDS: A CASE REPORT WITH FOCUS ON RADIOGRAPHIC APPERANCE
}

\author{
Mazen A. Aldosimani
}

\begin{abstract}
Cribriform adenocarcinoma of minor salivary gland (CAMSG) is currently classified as a subtype of polymorphous adenocarcinoma. CAMSG differs from polymorphous adenocarcinoma in that it has a distinct histopathologic appearance and clinically CAMSG has higher rate of lymph node metastasis at initial presentation. This report describes the radiographic appearance of a case of CAMSG on panoramic radiograph, cone-beam computed tomography (CBCT), multi-detector computed tomography (MDCT) and on magnetic resonance imaging (MRI).
\end{abstract}

\section{INTRODUCTION}

In the head and neck region there are between 450 to 1000 minor salivary glands and the majority of these glands are located within the oral cavity and oropharynx ${ }^{(1)}$. Of all tumors that develops in the salivary glands $10 \%$ to $20 \%$ originate from minor salivary glands and only $2 \%$ to $4 \%$ of head and neck cancers develop in minor salivary glands ${ }^{(1,2)}$. The most common location for minor salivary glands tumors is the hard palate where $50 \%$ of these tumors are malignant ${ }^{(1)}$. The most common types of malignancy that develops in minor salivary glands are adenoid cystic carcinoma and mucoepidermoid carcinoma ${ }^{(3)}$. Both of these tumors could have a low-grade variant where it behaves less aggressively and have radiographic features that somewhat resembles benign neoplasms. They also have high-grade malignant variant that behave more aggressively that would have the radiographic appearance expected from a malignant neoplasm ${ }^{(1)}$.

Cribriform adenocarcinoma of minor salivary gland (CAMSG) is currently classified as a subtype of polymorphous adenocarcinoma ${ }^{(4)}$, CAMSG differs from polymorphous adenocarcinoma in that it has a distinct histopathologic appearance and clinically CAMSG has higher rate of lymph node metastasis at initial presentation ${ }^{(5)}$. Polymorphous adenocarcinoma is a rare malignant neoplasm that mostly develops in minor salivary glands (1) and has been considered in the past to be a low-grade malignancy which was named polymorphous lowgrade adenocarcinoma since the disease rarely metastasis ${ }^{(4)}$. However, due to increased observation of high recurrence rates, World Health Organization (WHO) have changed the nomenclature of the tumor to polymorphous adenocarcinoma and have

* Assistant Professor, Department of Oral Medicine and Diagnostic Sciences, Division of Oral and Maxillofacial Radiology, College of Dentistry, King Saud University, Riyadh, Saudi Arabia 
removed the "low-grade" out (4). Radiographically, polymorphous adenocarcinoma is most commonly encountered in the hard palate and even though it used to be considered a low-grade malignancy, it can cause bone destruction of the hard palate with medullary infiltration and can also invade blood vessels and nerves ${ }^{(1)}$.

Currently, there are no reports that describe the radiographic appearance of CAMSG and whether there is any difference between its radiographic appearance and polymorphous adenocarcinoma since their clinical behavior is different. This report describes the radiographic appearance of a case of CAMSG on panoramic radiograph, cone-beam computed tomography (CBCT), multi-detector computed tomography (MDCT) and on magnetic resonance imaging (MRI).

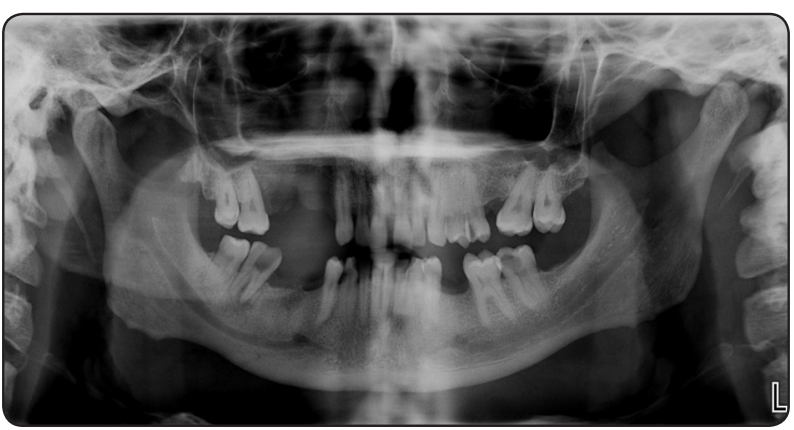

Fig. (1) Panoramic radiograph

\section{Case report:}

The patient is a 44 years old male who was complaining of pain in the right maxilla that started 8 months ago. The patient is smoker and reports that he had multiple extractions some involved the right maxilla. Clinical examination shows a large irregular mass with erythematous margins in the right maxilla at the premolar and first molar area. Right submandibular and left cervical lymph nodes were palpable and non-tender. Medical history was not relevant.

Panoramic radiograph shows an ill-defined radiolucent lesion in the right maxilla that extends from the distal of maxillary right canine to the mesial of maxillary second molar. The lesion has invasive borders and causes destruction and loss of the lamina dura and periodontal ligament space in the mesial of the second molar and distal of the canine. The lesion has also invaded into the right maxillary sinus destroying the floor of sinus.

CBCT images show a low attenuation lesion involving the right maxillary alveolar bone at the premolars and first molar area. The lesion has invasive borders and can be seen extending from the distal of the right maxillary canine to the mesial of the second molar causing destruction and loss of both buccal and palatal cortical plates and also extending superiorly involving the floor of the right maxillary sinus and invading within the sinus. The lesion does not involve the greater palatine foramina.

On MDCT images, an irregular mass of soft tissue density is seen in the right maxilla, the lesion has slightly higher attenuation compared to adjacent muscles. The lesion involves the palate up to the midline and extending laterally causing destruction of the right maxillary alveolar process, the lesion borders are ill-defined. Areas of bone remnants can be seen within the lesion. After administration of contrast medium, no change is seen in the lesion and the lesion borders are still difficult to discern. Lymph nodes appear within normal and their appearance does not change after contrast medium administration.

On MR T1 weighted images the lesion has a signal that is isointense to adjacent muscles and the lesion cannot be differentiated from neighboring tissues, the internal structure of the lesion is homogenous. After administration of contrast medium, on T1w images with fat suppression, the lesion had higher signal intensity compared to adjacent muscles and most of its borders can be clearly identified, the internal structure of the lesion is homogenous. The lesion dimensions were $2.1 \mathrm{~cm}$ mediolateraly, $2.5 \mathrm{~cm}$ superior-inferiorly and $3.2 \mathrm{~cm}$ anteroposteriorly. On T2w images with fat suppression, the lesion had a signal intensity higher than adjacent muscles and most of its borders can be clearly demarcated. 


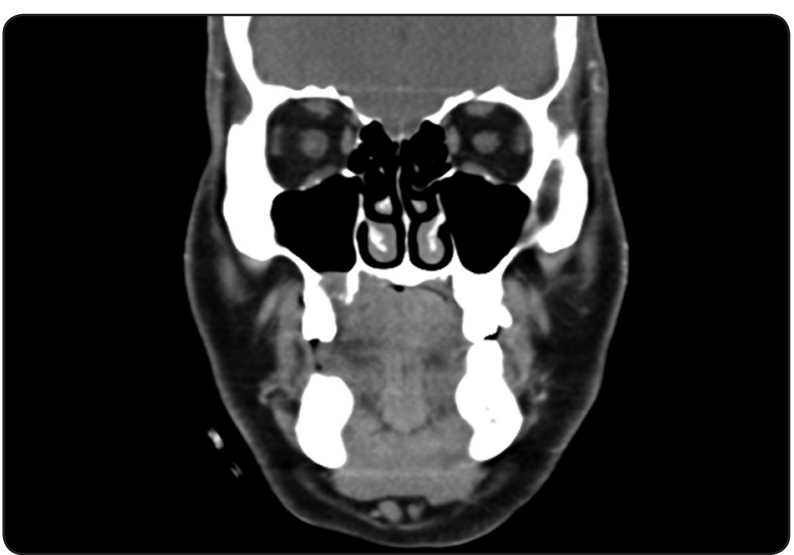

Fig. (2) MDCT coronal image with contrast medium enhancement

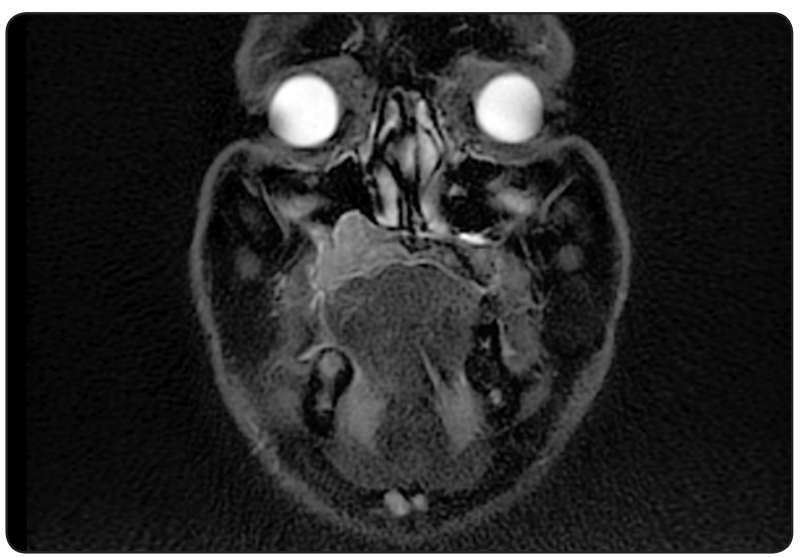

Fig. (3) MRI T2w coronal image

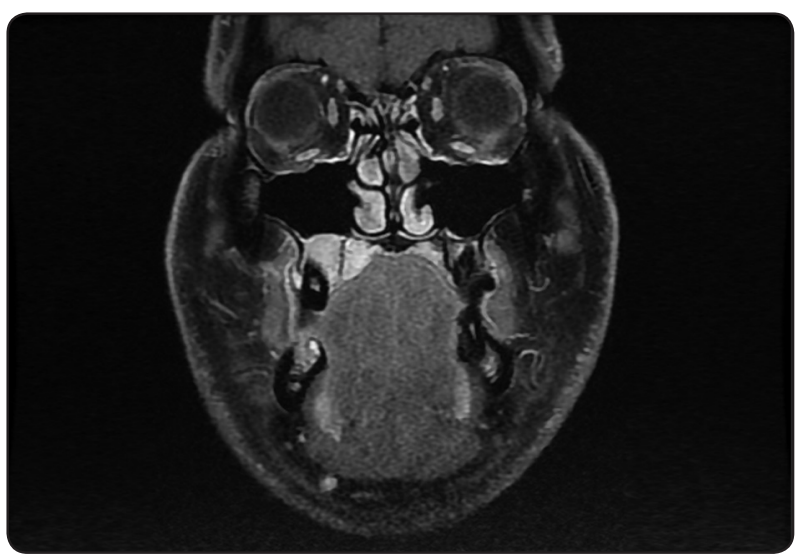

Fig. (4) MRI T1w coronal image with contrast medium enhancement
Histologically, the lesion features generally are in agreement with polymorphous adenocarcinoma but because of pronounced papillary architectural pattern and pseudopapillary projections it is morphologically suggestive of cribriform adenocarcinoma of minor salivary gland.

\section{DISCUSSION}

Differentiation between low-grade minor salivary glands malignant neoplasms and benign neoplasms radiographically is sometimes difficult because low-grade malignancies have indolent behavior and they appear similar to benign tumors on $\mathrm{CT}$ and MRI ${ }^{(6)}$. In the current case, the tumor had caused bone destruction of the right maxillary alveolar process, invaded into the maxillary sinus and had poorly defined invasive borders which may indicate the tumor is a high-grade malignancy. These radiographic features can be seen in high-grade tumors of adenoid cystic carcinoma and mucoepidermoid carcinoma ${ }^{(1)}$. Although polymorphous adenocarcinoma was considered a low-grade tumor in the past, it has been known to cause bone destruction with invasive borders in advanced tumors ${ }^{(1)}$.

The homogeneity of the tumor internal structure on CT and MR images can hint on whether the tumor is an aggressive tumor or low-grade tumor ${ }^{(6)}$. In general, aggressive tumors have heterogeneous appearance and this is also true after contrast medium enhancement on both CT and MR images ${ }^{(6)}$. In the current case, the tumor has shown a homogenous internal structure both before and after contrast medium enhancement on both $\mathrm{CT}$ and MR images which in general is similar how a low-grade malignancies and benign tumors would appear ${ }^{(6,7)}$.

The signal intensity of salivary gland tumors can indicate the tumor composition and tumors with a greater mucoid and serous content show low signal intensity on $\mathrm{T} 1 \mathrm{w}$ images and high signal intensity on T2w images which is seen commonly in low-grade malignant tumors and benign tumors ${ }^{\left({ }^{6}\right)}$. High-grade 
malignancies on the other hand show low signal intensity on both $\mathrm{T} 1 \mathrm{w}$ and $\mathrm{T} 2 \mathrm{w}$ images ${ }^{(6)}$. In the current case, the tumor shows low signal intensity on T1w images and high signal intensity on T2w images which is similar to low-grade malignancies and benign tumors.

The lesion borders were ill-defined on the panoramic radiograph, CBCT and MDCT images even after contrast medium enhancement. On MRI T1w images, the lesion was indistinguishable from adjacent tissues, however, after contrast medium administration, most of the lesion borders were clearly demarcated. Zeng et al ${ }^{(7)}$ reports that on conventional MRI ill-defined lesion borders were significantly more common among low-grade malignancies compared to benign tumors in their study.

\section{CONCLUSION}

Further studies that assess multiple cases of CAMSG are necessary to understand the radiographic appearance of these lesions.

\section{REFERENCES}

1. Abdel Razek AAK, Mukherji SK. Imaging of Minor Salivary Glands. Neuroimaging Clin N Am. 2018;28(2):295-302.

2. Baddour Jr HM, Fedewa SA, Chen AY. Five- and 10-Year Cause-Specific Survival Rates in Carcinoma of the Minor Salivary Gland. JAMA Otolaryngol Neck Surg. 2016 Jan 1;142(1):67-73.

3. von Stempel C, Morley S, Beale T, Otero S. Imaging of palatal lumps. Clin Radiol. 2017;72(2):97-107.

4. Mimica X, Katabi N, McGill MR, Hay A, Zanoni DK, Shah JP, et al. Polymorphous adenocarcinoma of salivary glands. Oral Oncol. 2019;95(May):52-8.

5. Madhura M, Kumar B, Suma S, Sarita Y. Cribriform adenocarcinoma of minor salivary gland: A mimic of polymorphous low-grade adenocarcinoma. J Oral Maxillofac Pathol. 2016;20(3):536-9.

6. Lee YYP, Wong KT, King AD, Ahuja AT. Imaging of salivary gland tumours. Eur J Radiol. 2008;66(3):419-36.

7. Zheng Y, Xiao Z, Zhang H, She D, Lin X, Lin Y, et al. Differentiation between benign and malignant palatal tumors using conventional MRI: a retrospective analysis of 130 cases. Oral Surg Oral Med Oral Pathol Oral Radiol. 2018;125(4):343-50. 